\title{
Lower Limb Thrombosis after Tibialis Tendon Tenorrhaphy: Case Report and Literature Review
}

\author{
Gustavo Constantino de Campos* \\ Instituto Wilson Mello, Research and Study Center Rua José Rocha Bonfim, 214 Campinas, SP, Brazil
}

\begin{abstract}
Background: Rupture of the tibialis anterior tendon is a rare lesion and only few reports are found in the literature. Another not so rare condition is a thromboembolic event following a lower limb surgical procedure or immobilization period. Although there is general agreement that patients undergoing major hip or knee surgery benefit from prophylactic anti-thrombotic therapy, currently there are no unanimously accepted recommendations for thromboprophylaxis in patients with isolated lower limb injury or surgery.
\end{abstract}

Purpose: The aim of this study is to provide a case report and a critical review of publications on the matter.

Case Report: We herein report a case of a 38-year-old man, otherwise well, who sustained a small open wound anterior to the tibiotarsal joint caused by the tip of a knife. Ultrasound confirmed a complete anterior tibialis tendon rupture. Patient underwent to acute repair and was discharged with an ankle cast, with the guidance of full-time use. There was no recommendation of any type of mechanical or pharmacological thromboprophylaxis. Five days later he presented deep venous thrombosis. Treatment was conducted with enoxaparin ( $20 \mathrm{mg}$ daily) for 3 months.

Discussion: Rupture of the tibialis anterior tendon is decidedly rare and often neglected. Acute repair may be attempted, since delayed tendon reconstruction has had less favorable functional results. Deep venous thrombosis is a potentially life threatening condition that can occur after lower limb injury or immobilization period. We performed a literature review but we cannot draw definite conclusions, given the paucity of high quality studies. In conclusion, there is a need for further research with deeper assessment of thromboprophylaxis indications and contraindications.

Keywords: Thromboprophylaxis; Lower limb; Orthopedic surgery

\section{Introduction}

Rupture of the tibialis anterior tendon is a rare lesion and only few reports are found in the literature [1-7]. Most subcutaneous ruptures occur in the elderly with structural weakness of the tendons $[8,9]$, and the diagnosis is often delayed, since patient can maintain dorsiflexion of the foot by compensatory use of extensor digitorum longus and extensor hallucis longus [10]. Open tendon tears can occur in younger individuals and usually result from injury to the dorsum of the ankle and foot on a healthy tendon [11]. Its diagnosis can also be confusing in case of a single injured tendon. An acute open tear should be treated with tenorrhaphy $[6,7,10,12,13]$.

Another not so rare condition is a thromboembolic event following a lower limb surgical procedure or immobilization period. Although there is general agreement that patients undergoing major hip or knee surgery benefit from prophylactic anti-thrombotic therapy, there are no unanimously accepted recommendations for thromboprophylaxis in patients with isolated lower limb injury or surgery [14]. This is concerning, since deep venous thrombosis (DVT) is associated with significant morbidity and mortality.

We present a patient with a traumatic tibialis tendon lesion that sustained a deep venous thrombosis (DVT) after tendon repair. Furthermore, we performed a critical review of the literature about anterior tibialis tendon lesion as well as about thromboprophylaxis in orthopedic surgery.

\section{Case Report}

FM, 38 years old, male, otherwise well, sustained a small open wound (less than 1 centimeter) caused by the tip of a knife. Patient said he was cooking one hour earlier when he accidentally dropped the knife and tried to prevent it from hitting the ground with his left foot. Physical examination revealed a clean transverse small cut anterior to the tibiotarsal joint with minor bleeding and virtually no pain. Patient maintained active dorsiflexion with some difficulty. Wound was sutured and patient referred to imaging. Ultrasound confirmed a complete anterior tibialis tendon rupture. The next day patient underwent to acute repair and was discharged with an ankle cast, with the guidance of full-time use. There was no recommendation of any type of mechanical or pharmacological thromboprophylaxis.

Five days later he presented calf pain and edema. The Doppler ultrasound showed calf deep venous thrombosis. Treatment was conducted with enoxaparin (20 mg daily) for 3 months. At 1-year follow-up patient was asymptomatic and fully recovered.

\section{Discussion}

The tibialis anterior muscle is the most medial muscle of the anterior compartment of the leg. It courses toward the medial border of the foot, inserting vertically on the first metatarsal base and the first cuneiform bone [11]. Combined with the extensor digitorum longus and extensor hallucis longus (EHL) muscles, the tibialis anterior muscle is responsible for dorsal extension of the ankle. Of these three muscles, most of the power contributing to ankle extension is attributed to

*Corresponding author: Gustavo Constantino de Campos, Instituto Wilson Mello Research and Study Center Rua José Rocha Bonfim, 214 Campinas, SP, Brazil, Tel: +551937089999; E-mail: gustavoccampos@usp.br

Received October 20, 2014; Accepted November 23, 2014; Published November 25, 2014

Citation: Campos GCD (2014) Lower Limb Thrombosis After Tibialis Tendon Tenorrhaphy: Case Report and Literature Review. J Trauma Treat 4: 222. doi:10.4172/2167-1222.1000222

Copyright: $\odot 2014$ Campos GCD. This is an open-access article distributed under the terms of the Creative Commons Attribution License, which permits unrestricted use, distribution, and reproduction in any medium, provided the original author and source are credited. 
the tibialis anterior muscle [15]. Although overuse tendonitis is not uncommon, rupture of the tendon is decidedly rare and often neglected.

Whatever the etiology, diagnosis of the lesion should be clinically established first. Accurate testing of the anterior tibialis muscle function is thus essential. The tibialis anterior tendon adjacent to the tibial crest should not be mistaken for the EHL tendon, which is thinner and associated with hyperextension of the big toe. It is essential to compare with contralateral side. If diagnosed early an acute repair may be attempted. Delayed tendon reconstruction has had less favorable functional results, including progressive retraction and muscle atrophy. After procedure, patient should be managed with immobilization in a short-leg cast with the ankle in $0^{\circ}$ of dorsiflexion for four to six weeks $[7,10]$.

Deep venous thrombosis (DVT) is potentially life-threatening condition that can occur after lower limb injury or immobilization period, but evidence-based recommendations for prophylactic treatment have not been established. The American Academy of Orthopedic Surgeons' (AAOS) clinical practice guideline about prevention of venous thromboembolic disease [16] only includes patients undergoing elective hip and knee arthroplasty. The Academy doesn't have a set of recommendations on thromboprophylaxis for any other type of orthopedic surgery, including trauma, lower limb arthroscopy or minor procedures. The International Consensus Statement for prevention and treatment of venous thromboembolism [17] recommend prophylactic treatment for all hip and knee replacements, hip fracture surgery and arthroscopic ligament reconstructions. Careful risk assessment should be undertaken for patients submitted to simple arthroscopy, isolated below knee injuries and plaster casts. The American College of Chest Physicians latest guideline [18] suggest no thromboprophylaxis for patients with isolated lower-extremity injuries requiring leg immobilization and for patients undergoing knee arthroscopy without a history of venous thromboembolism (grade 2B recommendation, which means it is not based on high quality studies). It also recommend against Doppler ultrasonography screening before hospital discharge.

Nilsson-Helander et al. [19] found a high incidence of DVT in patients with an acute Achilles tendon rupture that were treated either surgically or non-surgically, with no difference between groups. However, authors chose to screen all patients regardless symptoms, which certainly led to the high DVT incidence. Lassen et al. [20] demonstrated that thromboprophylaxis using LMWH (Reviparin) reduced the risk of DVT in patients treated with a below-the-knee cast for lower leg injuries in a study based on unilateral venography. Lapidus et al. [21] performed a randomized, placebo-controlled study on 105 consecutive patients, who were treated surgically for Achilles tendon rupture. Color duplex sonography was used as screening and, although the patients received thromboprophylaxis with 5,000 $\mathrm{U}$ of dalteparin daily during a 6-week period of immobilization, a DVT incidence of $34 \%$ was reported and no beneficial effect of thromboprophylaxis could be shown in comparison with placebo. Again, ultrasound DVT instead of symptomatic DVT as outcome could be a study limitation. Nevertheless, this study leads to a very interesting conclusion: it seems that immobilization after surgical procedure plays a great role in the DVT incidence, if not greater than the surgical procedure itself.

The present study has some limitations. First, it is only a case report. There is a need for prospective, randomized, clinical trials on proposed approaches with bigger samples and long-term results. Second, we performed a literature review but we cannot draw definite conclusions, given the paucity of high quality studies. In conclusion, this case reports two often under diagnosed conditions. Moreover, incidence of DVT after below-knee isolated injures or immobilization period is probably greater than we think. Current clinical guidelines do not yet unridle this important and potentially hazardous problem. There is a need for further research with deeper assessment of thromboprophylaxis indications and contraindications.

\section{References}

1. Michels F, Van Der Bauwhede J, Oosterlinck D, Thomas S, Guillo S (2014) Minimally invasive repair of the tibialis anterior tendon using a semitendinosus autograft. Foot Ankle Int 35: 264-271.

2. Palmanovich E, Brin YS, Laver L, Ben David D, Massrawe S, et al. (2013) Chronic tibialis anterior tendon tear treated with an Achilles tendon allograft technique. Orthopedics 36: 850-853.

3. Stapleton JJ (2013) Simultaneous surgical repair of a tibialis anterior tendon rupture and diabetic charcot neuroarthropathy of the midfoot: a case report. Clin Podiatr Med Surg 30: 599-604.

4. Yasui $Y$, Takao M, Miyamoto $W$, Matsushita T (2013) Reconstruction using an autograft with near complete preservation of the extensor retinaculum for chronic tibialis anterior tendon disruption. Arch Orthop Trauma Surg 133: 1669-1673.

5. Kontogeorgakos V, Koutalos A, Hantes M, Manoudis G, Badras L, et al. (2013) Traumatic tibialis anterior tendon rupture: treatment with a two-stage silicone tube and an interposition hamstring tendons graft protocol. Knee Surg Sports Traumatol Arthrosc.

6. Aderinto J, Gross A (2011) Delayed repair of tibialis anterior tendon rupture with Achilles tendon allograft. J Foot Ankle Surg 50: 340-342.

7. Negrine JP (2007) Tibialis anterior rupture: acute and chronic. Foot Ankle Clin 12: $569-572, \mathrm{v}$

8. Neumayer F, Djembi YR, Gerin A, Masquelet AC (2009) Closed rupture of the tibialis anterior tendon: a report of 2 cases. J Foot Ankle Surg 48: 457-461.

9. Patten A, Pun WK (2000) Spontaneous rupture of the tibialis anterior tendon: a case report and literature review. Foot Ankle Int 21: 697-700.

10. Sammarco VJ, Sammarco GJ, Henning C, Chaim S (2009) Surgical repair of acute and chronic tibialis anterior tendon ruptures. J Bone Joint Surg Am 91 325-332.

11. Lefebvre B, Beldame J, Bertiaux S, Biga N (2011) Open and subcutaneous recent tibialis anterior tendon ruptures: does postoperative immobilization method influence outcome? Orthop Traumatol Surg Res 97: 211-216.

12. Trout BM, Hosey G, Wertheimer SJ (2000) Rupture of the tibialis anterior tendon. J Foot Ankle Surg 39: 54-58.

13. Rodrigues ME, Pereira A, Alpoim B, Geada JM (2013) Rotura traumática do tendão tibial anterior - caso clínico. Revista Brasileira de Ortopedia 48: 278-281.

14. Struijk-Mulder MC, Ettema HB, Verheyen CC, Büller HR (2010) Comparing consensus guidelines on thromboprophylaxis in orthopedic surgery. J Thromb Haemost 8: 678-683.

15. Goetz J, Beckmann J, Koeck F, Grifka J, Dullien S, et al. (2013) Gait analysis after tibialis anterior tendon rupture repair using Z-plasty. J Foot Ankle Surg 52: 598-601.

16. Mont MA Jacobs JJ, Boggio LN, Bozic KJ, Della Valle CJ, et al. (2011) Preventing venous thromboembolic disease in patients undergoing elective hip and knee arthroplasty. J Am Acad Orthop Surg 19: 768-776.

17. Cardiovascular Disease Educational and Research Trust; Cyprus Cardiovascular Disease Educational and Research Trust; European Venous Forum; International Surgical Thrombosis Forum; International Union of Angiology; Union Internationale de Phlébologie (2006) Prevention and treatment of venous thromboembolism. International Consensus Statement (guidelines according to scientific evidence). Int Angiol 25: 101-161.

18. Falck-Ytter Y, Francis CW, Johanson NA, Curley C, Dahl OE, et al. (2012) Prevention of VTE in orthopedic surgery patients: Antithrombotic Therapy and Prevention of Thrombosis, 9th ed: American College of Chest Physicians Evidence-Based Clinical Practice Guidelines. Chest 141: e278S-325S. 
Citation: Campos GCD (2014) Lower Limb Thrombosis After Tibialis Tendon Tenorrhaphy: Case Report and Literature Review. J Trauma Treat 4: 222. doi:10.4172/2167-1222.1000222

Page 3 of 3

19. Nilsson-Helander K, Thurin A, Karlsson J, Eriksson BI (2009) High incidence of deep venous thrombosis after Achilles tendon rupture: a prospective study. Knee Surg Sports Traumatol Arthrosc 17: 1234-1238.

20. Lassen MR, Borris LC, Nakov RL (2002) Use of the low-molecular-weight heparin reviparin to prevent deep-vein thrombosis after leg injury requiring immobilization. N Engl J Med 347: 726-730.

21. Lapidus LJ, Rosfors S, Ponzer S, Levander C, Elvin A, et al. (2007) Prolonged thromboprophylaxis with dalteparin after surgical treatment of achilles tendon rupture: a randomized, placebo-controlled study. J Orthop Trauma 21: 52-57. 